\title{
EFFECTS OF CELECOXIB ON REACTIVE OXYGEN SPECIES PRODUCTION IN METASTASTIC MELANOMA CELLS
}

\section{Bruno A.L.A. Mariz, Ciro D. Soares, Jacks Jorge}

\begin{abstract}
This study evaluated the ROS production in B16F10 cells treated or not with a COX-2 inhibitor (celecoxib). After 24 hours with celecoxib treatment, B16F10 cells demonstrated an altered morphology, compatible with cell death process. The IC50 of this drug was $7.5 \mu \mathrm{M}$. In this dose, the treated groups demonstrated considerable reduction of the number of cells. A decrease in rates of cell proliferation and a significant reduction of ROS in celecoxib treated cells at their IC50 indicate this drug has cytotoxic activity, reduces ROS production and consequently is able to reduce cell proliferation. We concluded that celecoxib is an indicated drug for the treatment of aggressive melanomas.
\end{abstract}

\section{Key words:}

Melanoma, cell proliferation, reactive oxygen species.

\section{Introduction}

Human cells can produce byproducts of oxidative reaction known as Reactive Oxygen Species (ROS). When the synthesis of ROS is unbalanced, the cell's genetic material (DNA and RNA) can be damaged: genetic damage; proteins and lipids can also be affected: epigenetic damage ${ }^{1,2}$. These changes can module the expression of genes related to invasion and cell proliferation mechanisms. Neoplastic cells adapt their metabolism during the tumorigenesis process, and the production of ROSs is thought to be necessary for the invasion process ${ }^{3}$.

\section{Results and Discussion}

The cellular viability was assessed by 3-(4,5Dimethylthiazol-2-yl)-2,5-Diphenyltetrazolium Bromide (MTT) test. Damage to genetic material was analyzed by expression of bromodeoxyuridine (5-bromo-2'deoxyuridine, BrdU). The IC50 was determined using the software Compusyn ${ }^{\mathrm{TM}}$. The number of ROS positive cells was analyzed by flow cytometry.

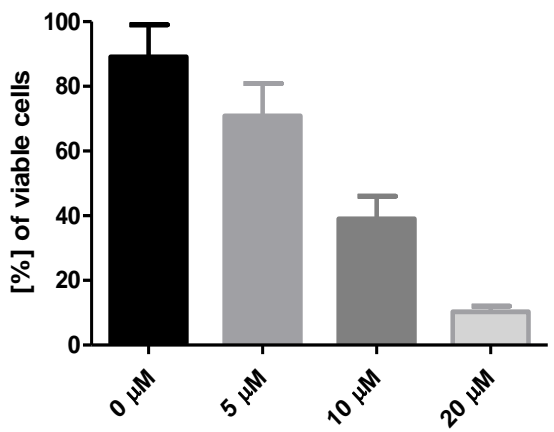

Image 1. Cytotoxic activity of celecoxib in B16F10 cells, MTT assay, and the graphic represents the percentage of viable cells after treatment.

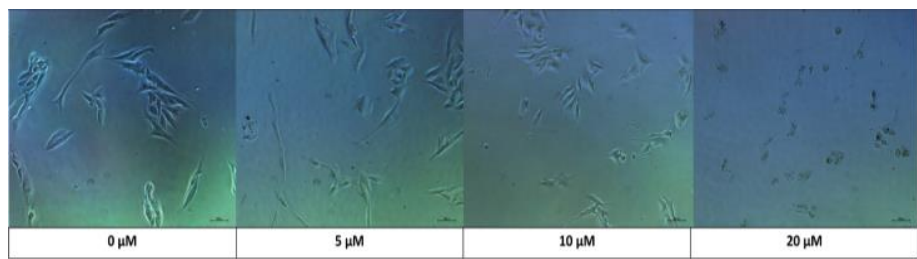

Image 2. B16F10 cells morphology after treatment with celecoxib.
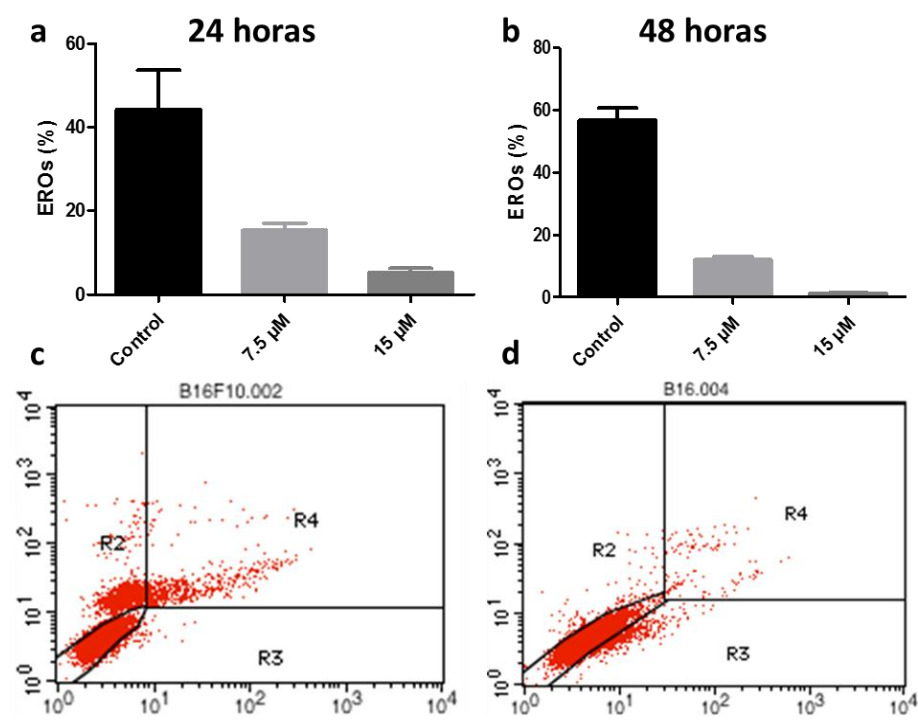

Image 3. ROS production after 24 (a) and 48 (b) hours of treatment with celecoxib (IC50). Significant DotPlot of control (c) and treated (d) groups (48 hours) analyzed in flow cytometer. In the control group (c) there is a greater number of cells that produced ROS (R2+ and R4+). However, in the treated group (d) there is a decrease in the R2+ e R4+ cells.

\section{Conclusions}

Celecoxib has cytotoxic activity, reduces ROS production and consequently is able to reduce cell proliferation, indicating that it could be used for the treatment of aggressive melanomas.

\section{Acknowledgement}

We would like to thank PIBIC-EM, CAPES and FAPESP for the financial support.

${ }^{1}$ Liu GS, et al. Proopiomelanocortin gene delivery induces apoptosis in melanoma through NADPH oxidase 4-mediated ROS generation. Free Radic Biol Med. 2014 May; 70:14-22.

${ }^{2}$ Quast SA, et al. Sensitization of Melanoma Cells for Death Ligand TRAIL Is Based on Cell Cycle Arrest, ROS Production, and Activation of Proapoptotic Bcl-2 Proteins. J Invest Dermatol. 2015 Nov;135(11):2794-804.

${ }^{3}$ Soares CD, et al. Prognostic significance of cyclooxygenase 2 and phosphorylated Akt1 overexpression in primary nonmetastatic and metastatic cutaneous melanomas. Melanoma Res. 201OJuñ 0.19146/pibic-2017-79093 XXV Congresso de Iniciação Científica da UNICAMP 